Cinémas

Revue d'études cinématographiques

Journal of Film Studies

\title{
Mélancolie et cinéma
}

\section{Denis Bellemare}

Volume 8, numéro 1-2, automne 1997

Cinéma et mélancolie

URI : https://id.erudit.org/iderudit/024747ar

DOI : https://doi.org/10.7202/024747ar

Aller au sommaire du numéro

Éditeur(s)

Cinémas

\section{ISSN}

1181-6945 (imprimé)

1705-6500 (numérique)

Découvrir la revue

Citer cet article

Bellemare, D. (1997). Mélancolie et cinéma. Cinémas, 8(1-2), 147-166.

https://doi.org/10.7202/024747ar

\section{Résumé de l'article}

Cet article désire établir ce qui fonde la trame intrinsèquement liée du travail du film et du travail de la mélancolie, en mettant en abyme trois niveaux de questionnement : sur le plan philosophique, quelle position du négatif le cinéma partage-t-il de notre connaissance du monde sous le couvert de la représentation du réel? sur le plan artistique, quel est l'enjeu au cinéma du double régime de présence et d'absence de sa représentation mimétique? et enfin, sur le plan spécifiquement cinématographique, en quoi le travail du visuel de la représentation, du narcissisme et de l'imaginaire recoupent-ils singulièrement la mélancolie? 


\title{
Mélancolie et cinéma
}

\section{Denis Bellemare}

\begin{abstract}
RÉSUMÉ
Cet article désire établir ce qui fonde la trame intrinsèquement liée du travail du film et du travail de la mélancolie, en mettant en abyme trois niveaux de questionnement: sur le plan philosophique, quelle position du négatif le cinéma partage-t-il de notre connaissance du monde sous le couvert de la représentation du réel? sur le plan artistique, quel est l'enjeu au cinéma du double régime de présence et d'absence de sa représentation mimétique? et enfin, sur le plan spécifiquement cinématographique, en quoi le travail du visuel de la représentation, du narcissisme et de l'imaginaire recoupentils singulièrement la mélancolie?
\end{abstract}

\section{ABSTRACT}

This article seeks to establish the basis for the framework which is intrinsically linked to the work of the film and the work of melancholy by holding in suspense three levels of interrogation. On the philosophical level, what relation to the negative does cinema share with our knowledge of the world in its representation of the real ? On the artistic level, what is at stake for cinerna under the dual regime of presence and absence in its mimetic representation? Finally, on the specifically cinematographic level, in what way do the work of the visual in representation, of narcissism and the imaginary intersect with melancholy?

Après les nombreuses réflexions poursuivies dans les articles antérieurs, le lecteur aura compris la multiplicité des voies d'entrée de la mélancolie au cinéma. Avec l'abondance de ces diverses voies d'entrée, il aura, pour conclure, la légitimité de se poser 
encore une fois la question initiale, celle de la pertinence, voire de l'essence, de ce concept au regard du cinéma. En quoi ce concept, ou ce thème, ou cet affect, s'appliquerait-il mieux que l'amour ou l'envie par exemple aux différents aspects du cinéma, et en quoi épouserait-il mieux le cinéma plutôt qu'une autre forme d'expression artistique? Et qui plus est rétorqueront certains lecteurs, d'un point de vue strictement méthodologique, la tendance de tels projets d'études alliant arts et sciences humaines n'a-t-elle pas parfois la séduisante faiblesse de trouver justement ce que les auteurs cherchent, et ce mille fois plutôt qu'une? Et alors tout devient mélancolie au cinéma, et la tautologie vit de sa propre récurrence, de sa grande évidence.

Mais avant toute chose peut-on parler de la mélancolie comme n'étant qu'un thème, un concept, un affect? Lhistoire de la mélancolie témoigne à nos yeux d'un apport plus grand, plus fondamental et plus structurant de l'histoire de la pensée en Occident que ces diverses expressions ne le suggèrent. L'idée de thème n'aurait de souci que de couvrir un éventail de contenus d'affects identifiables à certains types de symptômes et décrivant la mélancolie comme pathologie, et ce suivant une variété de points de vue cliniques. Cette approche limiterait toute étude de film à une analyse des dispositions socio-psychiques à la mélancolie quant au contenu filmique et n'atteindrait en rien le coeur, le réseau des formes d'expression de la mélancolie spécifiques au langage cinématographique. Nous voulons plutôt ici arrimer une position, une posture permettant de définir la liaison intrinsèque entre cinéma et mélancolie. Le terme de concept ne peut non plus satisfaire à nos exigences, car il risque d'être entendu comme modèle, or nous réservons ce terme de concept à une chaîne de structures mettant en relation diverses procédures permettant de se faire rencontrer en tout ou en partie le travail de la mélancolie et le travail du film. La mélancolie n'agira pas envers le cinéma uniquement comme thème, mais bien comme structure et concept, comme véritable opérateur.

Sans vouloir faire une synthèse de l'histoire de la mélancolie en Occident, il m'apparaît essentiel de relever deux questions, de tracer dans cette longue introduction une manière de premier cercle comportant deux grandes configurations (souvent appe- 
lées et rappelées dans les articles précédents) à partir duquel se détache et se dessine la spécificité de notre questionnement à l'intérieur de cet article.

\section{Premier cercle de deux grandes configurations}

Notre première question provient de la théorie hippocratique des quatre humeurs (la bile noire pour la mélancolie, le flegme, les biles jaune ou rouge pour la colère et le sang). Comment se fait-il que la bile (kholê) noire (melas), et par le fait même le tempérament mélancolique qui lui a été associé, constitua parmi toutes les humeurs l'objet d'études philosophiques et psychologiques le plus soutenu jusqu'à nos jours, et ce en dualité contrastante avec une autre humeur, celle de la bile rouge, celle du sang, de la jeunesse, de la passion, de la vie?

Notre deuxième question relance l'historicité de notre problématique, mais dans le champ particulier des arts et de la modernité. Comment expliquer, à la fin du XIX siècle et en ce $\mathrm{XX}^{\mathrm{e}}$ siècle, la correspondance de la modernité et de la prolifération des essais sur la mélancolie en littérature, en esthétique de l'art, en peinture, en photographie, au cinéma?

À la première question, Raymond Klibansky, Erwin Panofsky et Fritz Saxl, dans leur ouvrage monumental intitulé Saturne et la Mélancolie, comprennent dès l'origine «[...] que le problème particulier de la mélancolie [a] fourni le levain qui allait permettre le développement ultérieur de l'humorisme" (p. 44). Car la singularité de la bile noire résulte du fait qu'elle possède une " [...] propriété qui fait défaut aux autres humeurs : celle d'influencer la disposition de l'âme" (p. 76). Cette influence va de l'obscurcissement jusqu'à l'exacerbation de la conscience de soi, et cette disposition va devenir l'ambivalence constitutive même de la mélancolie jusqu'à nos jours. De la fureur chez Platon à la relation singulière et spirituelle qu'établit Aristote dans la mélancolie entre le génie et la folie, le concept polarise constamment ces oppositions jusqu'à la Renaissance, oppositions traduites aujourd'hui en termes de "conflits ambivalentiels» avec une topique et une dynamique duelle de retournement et de renversement des pulsions de vie et des pulsions de mort chez les freudiens et les post-freudiens. L'histoire de la mélancolie entraîne 
certainement une définition spécifique des cultures et des civilisations, mais plus essentiellement encore, elle nous invite à réfléchir sur les fondements actuels de notre système de pensée, de perception et de représentation, elle nous invite à nous attarder sur ce lot de bile noire présent depuis l'Antiquité grecque dans la posture mélancolique et qui insuffle du négatif dans la théorie de l'harmonie, de la symétrie des quatre humeurs, des quatre tempéraments, des quatre éléments, des quatre saisons. Car, qu'en est-il de ce négatif dans la constitution de notre compréhension de soi, des choses et du monde, dans notre conceptualisation et notre représentation du réel ? Et c'est peut-être dans ce sens d'une continuité philosophique mêlée à un profond désir de rupture qu'il faut comprendre le texte de Serge Cardinal, « La mélancolie du nom ", où à l'origine de la signification se trouve la négativité, dans cette impossibilité de concevoir autrement que du divisé et de l'opposé dans ce qui est multiple et différent. Et c'est aussi dans ce sens que dans le livre dirigé par Arthur Kleinmann et Byron Good, intitulé Culture and Depression, les textes gravitent autour d'une structure centrale et fondamentale d'un travail de deuil comme travail de culture. Il ne faut pas ici confondre ce travail du négatif dans la pensée et la culture avec le "négativisme social" où tout ce qui se nomme un groupe, une nation tombe dans le culturalisme et le narcissisme des petites différences. Au contraire du négativisme, la négativité signifie, détermine. Elle affirme, en une duplicité positivement négative, l'identité dans la diversité, la dualité dans l'opposition, le conflit dans la contradiction. Guy Rosolato reconnaît dans et par la négativité la médiation fondamentale et la dynamique la plus féconde dans le rapport à l'Autre et à l'inconnu (p. 10).

Si cette posture philosophique de la mélancolie comme travail du négatif nous aide à marquer en toile de fond un certain territoire introduit par notre illustrer question, il faut maintenant se reposer notre deuxième question, celle sur les arts et la modernité par rapport à l'effet d'entraînement mélancolique ou plutôt enfermés dans cette posture mélancolique perceptible dans plusieurs essais esthétiques contemporains. Dans l'histoire des cultures et des civilisations y a-t-il des périodes plus propices à l'expression mélancolique? 


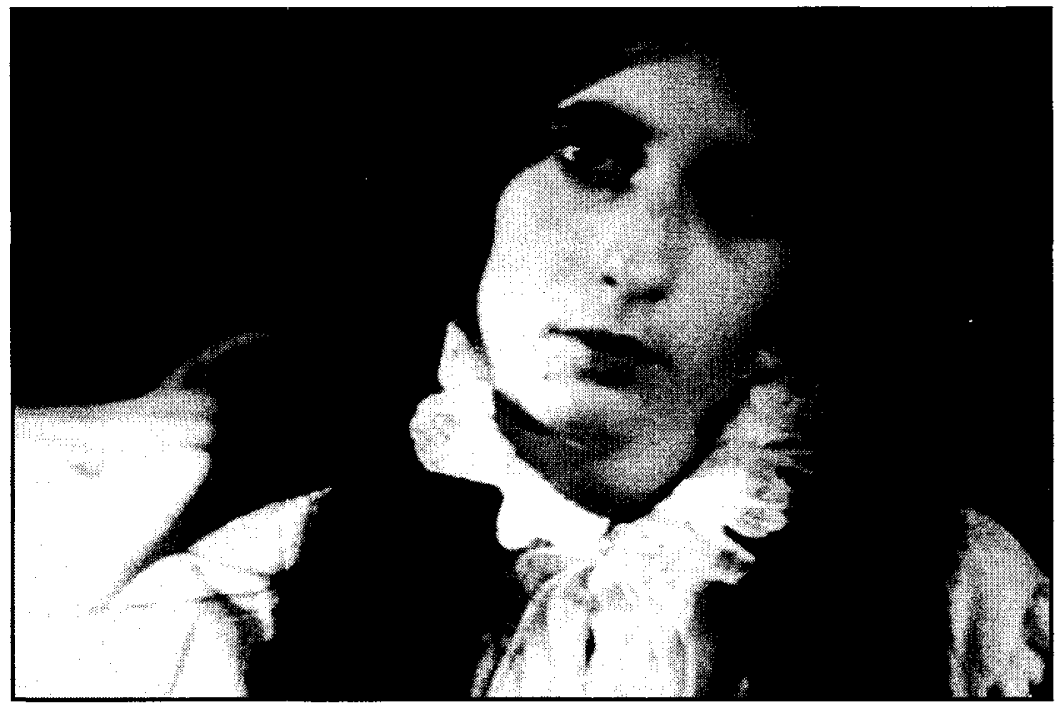

\section{Amour de perdition/Amor de perdicao de Manuel de Oliviera (1978)}

Collection Cinémathèque québécoise

Julia Kristeva s'interroge à partir du livre Origine du drame baroque allemand de Walter Benjamin sur ces déplacements des figures dans la traversée culturelle des affects mélancoliques dans le malaise des civilisations. Il faut se rappeler l'existence du conflit ambivalentiel de l'humeur mélancolique oscillant entre l'obscurcissement de la conscience et son exacerbation. Dans le drame baroque, l'allégorie confere un plaisir signifiant au signifiant perdu, mais que se passe-t-il quand l'expérience subjective de la mélancolie a difficulté à se nommer et n'a pas accès à cette dite jouissance?

Selon Walter Benjamin, c'est l'allégorie puissamment utilisée par le baroque et en particulier par le Trauerspiel (littéralement: jeu du deuil, jeu avec le deuil; dans l'usage: drame tragique du baroque allemand), qui réalise au mieux la tension mélancolique. [...] Il s'agit bien du sentiment mélancolique susceptible d'être nommé, mais que dire de la perte de l'objet et de l'indifférence au signifiant chez le mélancolique (Kristeva, 1987, p. 113)? 
La "parure mélancolique», comme l'exprime Christine BuciGlucksman à propos de l'Âge baroque, se déleste historiquement peu à peu de ses satifactions de déréliction pour succomber sous le poids de l'objectivation, de la neutralisation, à son destin moderne. Elle observe, perplexe: «La modernité ne nâ̂trait-elle pas d'un tout autre œil de la pensée qui " regarde le négatif en lui et séjourne en lui " comme l'écrira Hegel?" (p. 25). Kristeva parle alors de "construction écran contre la perte" (1987, p. 62) dans ces époques qui voient s'écrouler idoles religieuses et politiques. Nicolas Abraham et Maria Torok relèvent dans leur texte Deuil ou Mélancolie ce caractère annulatoire du langage de la figure, sa tendance à la neutralité, à la démétaphorisation (p. 261). Tous ces écarts entre la mélancolie nommée et innommable, figurée et littérale, nous les retrouvons tout au long de ce numéro entre les théories de Benjamin et de Kristeva, entre les pratiques cinématographiques de Visconti, de Godard, de cinéastes belges, québécois. Et structurellement, ces oppositions semblent s'inscrire dans la définition même de la mélancolie au XVI ${ }^{\mathrm{e}}$ siècle d'après Marsilio Ficino: "C'est à l'un ou l'autre extrême que la mélancolie exerce son influence, comme si la nature ne faisait qu'une en sa stabilité et fixité. Voilà en tout cas un caractère extrême qui n'est pas le lot des autres humeurs" (Klibansky et al., p. 80).

Oui, il y a une traversée culturelle différente des affects mélancoliques dans l'histoire des civilisations, mais elle semble se traduire dans la modernité par un double de négation. Faut-il y voir dans le domaine des arts visuels un changement total de paradigme tel qu'exprimé dans Vie et mort de l'image de Régis Debray, où la représentation illusoire de l'image vue fait place à la simulation numérique d'une image visionnée et où la difficulté du figural va alors de pair avec la difficulté d'inscription de sa perte?

Ce premier cercle de deux grandes configurations historiques ouvre une vaste perspective d'interprétations, son esquisse a voulu diriger la sensibilité du lecteur vers cette tension philosophique et esthétique nécessaire à l'élaboration et à la compréhension de notre deuxième cercle de configurations spécifiques à l'expression de l'art et de la mélancolie avant de parvenir au noyau central de cet article sur la mélancolie et le cinéma. 
Deuxième cercle de configurations : mais de quelles mélancolies de l'art?

Cette union de la notion purement médicale de mélancolie et le concept platonicien de fureur trouva son expression dans ce qui, pour les Grecs, constituait une thèse paradoxale, à savoir que non seulement les héros tragiques, comme Ajax, Héraclès, et Bellérophon, mais tous les hommes véritablement hors du commun, que ce fût dans le domaine des arts ou dans ceux de la poésie, de la philosophie, ou de la politique - sans oublier Socrate et Platon - étaient des mélancoliques (Klibansky et al., p. 48).

Cette présence de la mélancolie et de l'art, ce transfert quasi permanent entre le domaine de la maladie et celui de l'esprit se retrouvent tout le long de l'histoire de la mélancolie dans l'expression d'un désir d'anéantissement de soi lié à la perte d'un idéal et à cette impossibilité perpétuelle pour un sujet de faire le deuil de l'objet perdu. Elisabeth Roudinesco et Michel Plon dans leur Dictionnaire de la psychanalyse retracent ce "tempérament mélancolique" chez les grand mystiques, toujours menacés de s'éloigner de Dieu, chez les révolutionnaires, toujours en quête d'un idéal qui se dérobe, et chez certains créateurs, toujours à la recherche d'un dépassement de soi (p. 661-664).

Sarah Kofman nous fait gravir un échelon précieux en délaissant le mode individuel de l'expression mélancolique chez les créateurs pour le retourner au mode discursif de l'art. La mélancolie de l'artiste s'imbrique dans la structure même de la Mélancolie de l'art, titre de son livre. Au-delà du langage univoque de la science et de la technologie voulant subordonner l'art au logos, à la vérité, l'art joue sur deux tableaux à la fois, car au moment où il se montre présent par la mimesis de sa représentation, il se révèle comme n'étant pas d'ici tant son objet du monde est voué à la disparition: "Le pathos "paradoxal" que suscite "l'art" est l'envers de son essence paradoxale, celle-là même du "double", de ce colossos substitut du "cadavre absent" "(p. 17). Plus profondément encore, l'essai de Sarah Kofman entame l'idée de beauté dans l'art qui, selon elle, n'est jamais exempte de mélancolie: "Avec l'art, il n'y va pas d'un simple travail du négatif, 
mais d'un travail de deuil irrelevable par quelque dialectique maîtrisante" (p. 20). Les hypothèses de Julia Kristeva et de Pierre Fédida abondent aussi dans ce sens et ont recours au texte de Freud de 1915-1916, "Éphémère Destinée", pour relier à la perte de l'objet dans le deuil l'éphémère et le beau, la sublimation et l'art. Julia Kristeva évoque l'art comme emprise sublimatoire sur la chose perdue et cette dynamique se tisse autour du vide dépressif (1987, p. 109-111). Tout en redoublant cette rencontre de l'éphémère et du "beau objet ", Fédida retient de ce texte de Freud une plus grande violence face à ce temps de rareté et pousse à l'avant-scène «[...] la révolte de l'âme contre le deuil qui a dévalorisé chez eux la jouissance du Beau» (Freud, 1988, p. 233-236).

La beauté - que la sublimation dépressive protège -
est pourtant une violence qui ne manque pas d'apparât-
tre dans le processus mélancolique. Et le deuil mélan-
colique ne concerne pas tant la destruction du bon
objet que la mise à mort de la beauté même (Fédida,
1978, p. 78).

D'un processus psycho-critique analysant la mélancolie de certains créateurs à une approche structurante signifiant la mélancolie même de l'art, nous franchissons une autre étape avec Marie-Claude Lambotte. Dans le recoupement de plusieurs types de discours et l'analyse de ses variantes, l'auteure propose à travers l'histoire de la mélancolie un réel projet esthétique: la mélancolie de l'art et l'esthétique de la mélancolie s'arriment partiellement à une identité de structures. Du mélancolique, quelle faille originaire le sujet s'épuise-t-il à combler sans jamais y parvenir, et du regard dans le visuel de la représentation, quelle faille spéculaire le sujet doit-il surmonter quand sa propre identité lui a été ravie par un miroir aveugle? Et cette spécularité, dans cet enjeu même du visuel et de la représentation, conduit dans sa faillite à un regard perdu par l'absence d'éléments identificatoires, à un sentiment de vide dans l'impossibilité d'inscription du sens.

De même notre premier cercle de configurations de l'histoire de la mélancolie est venu nous dire la part du négatif comme 
médiation fondamentale dans la signification, la nomination, la représentation des objets du monde; de même notre deuxième cercle de configurations de la mélancolie et de l'art vient souligner non seulement un travail de deuil et de perte dans l'essence paradoxale de la représentation mimétique en art jouant sur le double régime de la présence et de l'absence, mais encore il exprime dans l'enjeu du visuel la problématique identitaire. Sous le couvert du travail du négatif, nous sommes maintenant en bonne position théorique pour retenir une structure opératoire inaugurale dans la rencontre de la mélancolie et du cinéma: la problématique de l'identité dans la perception et le visuel de la représentation.

\section{- NOTRE DÉFINITION DE LA MÉLANCOLIE}

À partir de ce moment que pouvons-nous retenir comme définitions de la mélancolie? C’est le pôle négatif de la bile noire dans les quatre humeurs, c'est le pôle des pulsions de mort en oppositon aux pulsions de vie en psychanalyse, c'est le non du oui dans la nomination et la perception des objets du monde, c'est le pôle de la fragilité du signifiant et de l'effondrement du sens en sémiotique.

Mais plus précisément encore, à partir du texte fondateur de Freud, Deuil et Mélancolie (1917), nous souhaitons livrer en premier lieu notre approche de la mélancolie afin de bien asseoir ce qui de son travail semble vouloir interpénétrer en fragments, en quartiers, en tangentes quelques aspects du langage cinématographique et en second lieu, nous voulons ressortir de réflexions théoriques sur le cinéma ce qui l'engage parfois dans des voies mélancoliques.

Plusieurs articles de ce numéro ont déjà bien décrit du texte de Freud les grands traits de ressemblance de la mélancolie avec le deuil. Mais je veux principalement attirer votre attention sur le fait que contrairement au deuil, le mélancolique méconnaît l'objet précis de sa perte, la réaction à la perte sollicite déjà les caractères particuliers d'une abstraction, d'une énigme, ce qui a été perdu glisse hors de la conscience.

Nous sommes en présence pour mieux cerner l'accès mélancolique d'un premier mouvement d'ouverture de la perte par le 
deuil et puis d'un second mouvement de fermeture dans le retour de cette perte sur le Moi. Cette ombre entame l'identité du Moi, l'affecte dans une zone totalement livrée aux antagonismes, aux dualités, à une expérience d'incomplétude qu'un troisième mouvement, par le retrait même de cette perte dans le Moi, engage dans la voie régrédiente du narcissisme (Freud, 1983, p. 158).

La mélancolie emprunte donc une première partie de ses caractères au deuil, mais sa seconde partie se constitue, avonsnous dit, comme une énigme par la capacité imaginaire du sujet à s'attacher la présence de l'objet, mais comme objet perdu (1983, p. 151).

La dynamique de la mélancolie en cette identification du Moi à l'objet abandonné se joue en des représentations nourries par tout un paradigme de négativités: perte, manque, abandon, absence, déni, dénégation. Il est essentiel de retenir qu'il s'agit bien ici de représentations.

Le sujet mélancolique doit pourtant recouvrir l'intolérable réel de cette perte, de ce manque, de cette absence, de cet abandon, et c'est par le processus de régression par identification narcissique qu'il maintiendra intact imaginairement la présence de l'objet perdu. L'identification narcissique entraînera une modification des liens signifiants digne de toute bonne représentation, et ce du réel à un régime imaginaire.

Comment s'étonner alors de la constitution paradoxale du sujet mélancolique? Paradoxal en effet ce clivage du sujet qui met en conflit un Surmoi sévère, lucide, observateur à la perception exacerbée, menaçant, porteur de complaintes et d'autoreproches et un Moi imaginairement investi à restaurer son idéal perdu. Tout aussi contradictoires sont les processus empruntés par le sujet mélancolique ouvrant imaginairement par la dénégation de la perte de l'objet une sorte d'issue compensatoire pour pouvoir, au bout de sa stupeur, dénier, résister à toute signification.

Ce parcours freudien et post-freudien prend une direction quelque peu différente par l'inclinaison métapsychologique que lui donne Pierre Fédida en définissant la mélancolie vers le rêve moins comme «[...] la réaction régressive à la perte de l'objet 
que la capacité fantasmatique (ou hallucinatoire) de le maintenir vivant comme objet perdu" (p. 76).

Le mélancolique, écrit Kristeva, est nécessairement un habitant de l'imaginaire (1987, p. 72). Dans les écrits de Freud et de Kristeva, ce qui peut étonner, ce sont les capacités de représentation et de production du travail de mélancolie. Ainsi, ces représentations et ces productions deviennent les marques communicables d'une réalité affective présente et sensible. Pour Kristeva, la création esthétique (p. 35) semble être une représentation sémiologique très fidèle de la lutte du sujet mélancolique contre l'effondrement symbolique. Les productions imaginaires semblant alors des solutions sublimatoires de ces crises.

Ce que nous pouvons fondamentalement retenir ici de ces théories psychanalytiques, c'est cette grande occupation, cette grande capacité de représentations du sujet mélancolique à maintenir imaginairement présent en lui, intact ce qui lui est réellement absent, manquant, perdu.

\section{- NOTRE APPROCHE THÉORIQUE DU CINÉMA}

Les apports théoriques déployés ici à circonscrire notre objet cinéma ont principalement une visée psychanalytique. À l'instar de J.-L. Baudry, nous sommes intéressés par ces connivences, correspondances et affinités spécifiques entre le dispositif cinématographique, nos facultés de représentation et le Moi-sujet de la psychanalyse (p. 10-11 et 24). Notre grande préoccupation sera de soulever les caractéristiques psychanalytiques du dispositif cinématographique, de son appareil de base, et de la posture spectatorielle singulière qu'elle induit.

Baudry insiste sur le caractère artificiel du ciné-sujet, pure production du dispositif cinématographique qui favorise cette construction et cette position. S'il fait de l'impression de réalité la clé de la représentation cinématographique (p. 37), ce n'est pas tant parce qu'elle redouble aussi exactement que possible le réel, mais c'est plutôt qu'en termes de simulation, cette représentation engage quelque chose que le spectateur aurait archaïquement connu et puis perdu. Le dispositif cinématographique enclencherait nostalgiquement la répétition de cet état archaïque où perceptions et représentations s'entremêlaient à la limite de 
l'indistinction. Ce dispositif déterminerait artificiellement cet état régressif par un retour vers un narcissisme relatif: retrouver cet état antérieur, mais maintenent perdu. Dès lors, il s'agit bien pour Baudry d'une simulation d'un état du sujet, d'une position du sujet, d'un effet sujet spécifique du cinéma et non de la réalité (p. 48).

Cette première base jetée, Christian Metz, dans le Signifiant imaginaire, verra à définir les articulations fines et complexes, les parentés partielles qu'entretiennent cinéma et psychanalyse, la visée filmique et divers régimes psychiques. Premièrement, le spectateur entretient aves les films de véritables relations d'objet (p. 12), relations fantasmatiques bien distinctes des relations réelles et des objets réels. Deuxièmement, il s'agit bien ici d'une relation imaginaire et troisièmement, cette relation imaginaire opère sur le mode de l'absence (p. 62-65).

Ces trois premières données de la théorie metzienne viennent qualifier la perception au cinéma d'un enjeu imaginaire assez troublant entre présence et absence allant au-delà d'une simple simulation d'impression de réalité. Tout est absent, tout est enregistré comme une trace mnésique sur l'écran du cinéma. Pour Metz, c'est le signifiant cinéma tout entier qui est absence (p. 63). Le cinéma nous engage dans l'imaginaire, mais avec ce caractère double, ambivalent, paradoxal qui le soutient. Il combine en lui une certaine présence par sa richesse perceptive inhabituelle et une certaine absence en frappant cette perception d'irréalité (p. 65), en la faisant basculer aussitôt dans sa propre absence. Et à son tour, le miroir second de l'écran reprend cet enjeu imaginaire sur le registre du reflet et du manque (p. 80). L'activité de perception au cinéma y est réelle, mais le perçu n'est pas l'objet, c'est son ombre, son fantôme, son double, sa réplique. Et en ce sens, les réflexions de Metz rejoignent celles de Sarah Kofman sur l'essence paradoxale de l'art, celle-là même du double jeu de présence et d'absence.

Le cinéma doit être compris ici dans cette grande continuité d'une histoire du regard en Occident, de cet « œil interminable» écrirait Jacques Aumont, et ce de la peinture jusqu'à la photographie. Le cinéma s'enligne donc dans la logique des essais théoriques, ci-haut nommés, sur la mélancolie et l'art en général. 
Toutefois son dispositif, comme nous l'expliquons dans ce souschapitre, semble ancrer et rencontrer davantage le travail de la mélancolie. L'essai d'Alain Buisine sur Eugène Atget ou la mélancolie en photographie abonde dans notre sens. Si toute l'ouvre d'Atget a partie liée avec la perte, la mort, la mélancolie, Buisine prolonge son questionnement sur ce que serait «[...] la mélancolie d'un créateur travaillant dans une discipline qui elle-même est par nature mélancolique» (p. 179). Pour lui, la perte est consubstantielle à la nature du geste photographique. Les photographies inscrivent la perte même dans ce qui ne risque jamais de disparaître. Il existe donc une négativité du geste photographique issue de l'imaginaire collectif d'une technique mimétique (p. 177181). Et c'est sous cet angle que nous vous invitons à poursuivre nos réflexions sur le dispositif cinématographique.

Dans cette emprise même du signifiant imaginaire du cinéma, le spectateur s'identifie primairement à lui-même comme pur acte de perception (Metz, p. 69). Cette position d'un Moi leurré au cinéma défie ainsi toute illusion de maîtrise perceptive, l'illusion en quelque sorte d'une plénitude de la relation d'objet que le spectateur tenterait d'établir avec le cinéma. Le régime scopique du cinéma vise à installer une nouvelle figure du manque, l'absence physique de l'objet vu. Et dans sa passion de percevoir (pulsion scopique, pulsion entendante), c'est ce manque que le spectateur veut combler, c'est ce manque qu'il a soin de toujours maintenir béant pour survivre comme désir (Metz, p. 83).

Ce que nous pouvons fondamentalement retenir ici de ces théories psychanalytiques, c'est la production, la construction d'un sujet spectatoriel par le dispositif cinématographique sous le couvert d'une régression narcissique tentant de rétablir imaginairement la plénitude d'une relation d'objet: relation d'autant plus paradoxale qu'elle opère sur le mode de l'absence.

\section{Cinéma et mélancolie}

De la surimpression de ces deux approches théoriques psychanalytiques sur la mélancolie et le cinéma, le lecteur peut maintenant mieux circonscrire ce qui vient du cinéma et va à la mélancolie, ce qui vient de la mélancolie et va au cinéma. Ce qui 
appartient à l'un et puis à l'autre ne circule jamais entièrement dans la même direction, mais jamais non plus dans le sens contraire, ces rencontres partielles et incomplètes, mais rarement divergentes, nous permettent de leur créer un espace commun de travail.

Dans notre deuxième cercle de configurations, nous avons été en mesure de retenir une structure opératoire inaugurale dans la rencontre de la mélancolie et du cinéma: il s'agissait de la problématique de l'identité dans la perception et dans le visuel de la représentation. Nous sommes maintenant en mesure de la compléter, cette structure, en spécifiant de cette problématique de l'identité son caractère narcissique et en déterminant de la perception et du visuel de la représentation son régime imaginaire, et ce toujours sous le couvert de la négativité. Nous pouvons alors rassembler les maillons nécessaires à notre chaîne du travail de la mélancolie et du travail du cinéma et les nommer et disposer ainsi: dans les processus de la représentation dans la mélancolie et du visuel de la représentation au cinéma perdure un écart du réel à combler dans la perception du sujet mélancolique et du sujet spectateur de cinéma et pour ce faire, pour restaurer la totalité de la satisfaction que le réel ne lui rend pas, le sujet par la voie régrédiente du narcissisme peut raviver et rendre présent imaginairement ce qui lui manque, ce qu'il a perdu, ce qui lui est absent.

\section{- DE LA REPRÉSENTATION ET DU VISUEL}

La fin première et immédiate de l'épreuve de réalité n'est donc pas de trouver dans la perception réelle un objet correspondant au représenté, mais de le retrouver, de se convaincre qu'il est encore présent (Freud, 1987, p. 138).

Nous avons constaté dans le travail de la mélancolie la très grande capacité du sujet à évacuer la réalité et à lui substituer une représentation plus satisfaisante. La perception du mélancolique part du réel pour aussitôt y projeter sa part manquante. Au cinéma, cette épreuve de réalité est beaucoup plus labile puisqu'il s'agit d'une impression de réalité de la représentation filmi- 
que, c'est ainsi que Baudry a pu dire que perceptions et représentations s'entremêlent au-delà d'une simple simulation de réalité. De cette étape, il faut retenir du visuel et de la représentation sa très grande manipulation du réel afin de combler un déficit perceptuel.

De même Kant nous a avertis de ne pas oublier que notre perception a des conditions subjectives et de ne pas la tenir pour identique avec le perçu inconnaissable, de même la psychanalyse nous engage à ne pas mettre la perception de la conscience à la place du processus psychique inconscient qui est son objet. La perception surgit toujours d'une quelconque élaboration qui explique ce décalage entre ce qui est dehors et ce qui est dedans, entre ce qui est et ce qui est représenté. Cette polarité instaure déjà l'ambivalence d'un premier monde qui entre projection et introjection, s'affirme et se nie. L'on retrouve ainsi à l'origine de la perception un travail du négatif qui tente, par la projection, de combler un déficit. La représentation comme acte, comme construction est issue d'un double mouvement, celui de la perception et de la projection, du réel et de l'imaginaire où, en termes économiques, l'on tente de se partager une vision régulatrice et formatrice du monde, où en termes dynamiques, l'on tente de se protéger à l'extérieur d'excitations pénibles.

Dès lors que je perçois, écrit Lacan, mes perceptions m’appartiennent: "Dans notre rapport aux choses, tel qu'il est constitué par la voie de la vision, et ordonné dans les figures de la représentation, quelque chose glisse, passe, se transmet, d'étage en étage, pour y être toujours à quelque degré éludé" (p. 70). C'est ça qui s'appelle le regard, le piège à regard, à perception, à représentation. Entre l'instauration d'un sujet et la restauration d'un objet, l'on est à même de voir la force de la négativité mise au travail dans le visuel.

\section{Dans L'CEil et l'esprit, Merleau-Ponty écrit ceci de l'image :}

Le mot d'images est mal famé parce qu'on a cru étourdiment qu'un dessin était un décalque, une copie, une seconde chose et l'image mentale un dessin de ce genre dans notre bric-à-brac privé. Mais si, en effet, elle n'est rien de pareil, le dessin et le tableau n'appartiennent pas plus qu'elle à l'en-soi. Ils sont le dedans du dehors

Mélancolie et cinéma 
et le dehors du dedans, que rend possible la duplicité du sentir, et sans lesquels on ne comprendra jamais la quasi-présence et la visibilité imminente qui font tout le problème de l'imaginaire (p. 23).

Le travail du visuel ne répond pas toujours à " [...] l'idéale complétude que proposent les dispositifs de représentation" (Didi-Huberman, p. 191). Limage est du domaine de l'incertitude et non pas du logos et de la vérité.

\section{- DU NARCISSISME}

La représentation mélancolique porte en germe non pas tant l'objet perdu en lui-même tel le ferait le deuil, mais plutôt l'image même de cet objet perdu renversée comme atteinte à l'image de la représentation du Moi. Ce processus de retournement et de renversement n'est possible que par la voie régrédiente du narcissisme. Au cinéma, le spectateur s'identifie primairement à lui-même comme pur acte de perception dans l'illusion en quelque sorte d'une plénitude de la relation d'objet à restaurer. Le spectateur de cinéma aime se percevoir, s'aime percevoir, il est pris en flagrant délit d'auto-amance de perception.

Le Moi est lié à l'image du corps propre. "L'image, c'est moi", s'écrierait Narcisse tel l'enfant au miroir lacanien. Le narcissisme est une question d'identification par l'image avant d'être l'amour ou la haine de cette image en soi de cette identité. "Le Moi n'est autre que cette captation imaginaire qui caractérise le narcissisme» (Nasio, p. 86). Comment ne pas se surprendre alors de la fascination de tout sujet pour les processus imageants?

Mais cette structure primaire, régrédiente, ouvre la lutte éternelle plus ou moins bien réussie entre ce désir de fusion et de distance par rapport à l'introjection de l'objet perdu. Car la nostalgie d'une toute-puissance d'un Moi à reconstituer va de pair avec une réalité déficiente sur le plan identitaire. Qui dit conflit, dit forces en présence, et dans le cas du sujet mélancolique et du sujet spectatoriel, elles sont paradoxales, ambivalentes et se promènent entre deux extrêmes.

La question du narcissisme est au cœur même des identifications formatrices de la fonction du Moi. Il y a donc, selon nous, 
une première identification primaire commune à tous les spectateurs de cinéma, celle du plaisir de se percevoir en train de percevoir, mais aussitôt cette base formée, elle est vite secouée, ébranlée, éclatée en de multiples identifications secondaires: ce sont différentes couches entremêlées des identifications où étrangement ces processus se recoupent, se doublent et se dédoublent sous différents régimes perceptuels du monde, de soi et du film, jamais tout à fait les mêmes, jamais tout à fait différents, mais gardant toujours la trace d'un manque, d'une incomplétude cette fois-ci spécifiques et secondaires, mais n'ayant de cesse de raviver une blessure narcissique originaire. Il n'y a donc pas une identification, il y a de l'identification avant toute chose dans des relations d'objet multiples sempiternellement retournées et renversées sur le Moi et ses images. Le Moi résulte de la sédimentation de ces investissements d'objets abandonnés. L'identification aux images de l'objet telle l'identification narcissique vise à restituer au sujet sa part manquante au nom d'un idéal.

L'article de Denise Pérusse dans ce numéro, "Le spectacle du "manque féminin" au cinéma: un leurre qui en cache un autre ", s'avère tout à fait éclairant. Sans dénier les spécificités et les différences des études féministes sur le cinéma, je ne peux m'empêcher d'y voir une métaphore, voire une définition générique de la spectature au cinéma. La place désignée de la spectatrice comme lieu d'une absence réelle ne peut que renvoyer en creux aux multiples enjeux de la présence et de l'absence induits par le dispositif cinématographique. Les écrits féministes disent aussi la variété des positions identificatoires: les femmes prennent-elles obligatoirement une position spectatorielle féminine et les hommes, une position masculine? La femme et sa relation privilégiée avec l'Imaginaire, la fluidité de son identité, ces positions multiples ne viennent-elles pas dire, à l'instar de Kaja Silverman, que tout sujet regardant est organisé autour de la même absence?

\section{- DE L'IMAGINAIRE}

Pourquoi le visible se prête-t-il à une synthèse primaire et fragile des pulsions, à une figurabilité plus souple,... 
plus risquée aussi? Le spéculaire est donc aussi le point de départ le plus précoce des signes, des identifications narcissiques et des transes fantasmatiques d'une identité parlante à une autre. Par ailleurs, dans ce nœud de frayeur et de séduction que leur propose le spéculaire, les hommes et les femmes se retrouvent différemment. Cette épreuve de la différence sexuelle comme celle de I'homosexualité, ce heurt avec nos identités impossibles jusqu'à la psychose, le cinéma n’arrête pas de les donner à voir (Kristeva, 1997, p. 134).

L'imaginaire n'est pas un reflet, écrit Julia Kristeva, mais une synthèse subjective. Non seulement soutient-t-elle, perplexe, que ce soit le "mal" qui structure l'imaginaire, mais encore que l'image cinématographique soit le lieu privilégié de la captation primaire de nos angoisses.

Le concept d'imaginaire ne vient-il pas dire à quelle enseigne se loge tout régime de perception et de représentation dans la circulation dynamique et duelle de forces antagonistes en présence? Le concept d'imaginaire ne vient-il pas signifier qu'il n'est pas l'imagination, mais bien l'image inconsciemment et défensivement altérée du réel afin de lui restituer une partie auparavant supprimée? Le concept d'imaginaire ne vient-il pas couvrir la duplicité positive du travail du négatif?

Le sujet mélancolique a cette grande capacité imaginaire à s'attacher la présence de l'objet mais comme objet perdu. Le spectateur de cinéma entretient avec les films de véritables relations d'objet, relations imaginaires qui opèrent sur le mode de l'absence. Mais plus paradoxalement, cet imaginaire du manque, comme nous l'avons vu, n'opère pas uniquement sous le mode du principe de réalité, mais encore sous le mode du principe de plaisir. Le sujet cherche sa satisfaction même dans sa complainte dérélictueuse.

Le manque est ce que le désir veut combler, et en même temps ce qu'il a soin de toujours maintenir béant pour survivre comme désir. Il n'a au fond pas d'objet, en tout cas pas d'objet réel, il poursuit un objet imaginaire (objet perdu) qui est son objet le plus véritable, un objet qui a toujours été perdu, et toujours désiré comme tel (Metz, p. 80).

La jouissance mélancolique et la jouissance-cinéma consistentelles en une véritable transgression imaginaire d'un manque? 
Fédida, dans L'Absence, ouvre une voie de la mélancolie vers le rêve et non pas vers le deuil comme l'a théorisé Freud. Cette satisfaction hallucinatoire du désir qu'est le rêve vient un peu, à la manière du signifiant imaginaire du cinéma dans le jeu de la présence et de l'absence de ses images, combler à la fois un manque et soutenir le véritable destin fantasmatique de cette relation d'objet comme objet perdu et comme "beau objet». Le fantasme de l'éphémère destinée et de l'éphémère beauté ne vient-il pas s'interposer entre la mélancolie et le rêve pour définir un autre pôle, en ceci conforme à la théorie des extrêmes de l'humeur noire, que celui ici démontré du travail de deuil et des angoissantes frayeurs? Des deux pôles, il n'en reste pas moins la même poursuite imagiraire d'un objet éternellement manquant. Cette espèce de chose-cinéma nous renvoie toutes et tous en anamorphose à la doublure comique et mélancolique du monde.

Il n'existe pas tout à fait en nous une disponibilité perceptive, mais une maladie du visible dont les images constituent l'étrange pansement, une application lénifiante, bien que nous ignorions sans cesse où se trouve la plaie vive, son oil et sa lumière ouverts dans le visible (Schefer, p. 198).

Université du Québec à Chicoutimi

OUVRAGES CITES

Abraham, Nicolas et Maria Torok. L'Ecorce et le Noyau. Paris: Aubier-Flammarion, 1978.

Baudry, Jean-Louis. L'Effet-cinéma. Paris: Albatros, 1978.

Buci-Glucksmann, Christine. "L'œil de la Pensée". L'Écrit du temps, n" 13 (1987), p. 23-44.

Buisine, Alain. Eugène Atget ou la mélancolie en photographie. Nîmes: Éditions Jacqueline Chambon, 1994.

Debray, Régis. Vie et mort de l'image. Paris: Gallimard, 1992.

Didi-Huberman, Georges. Devant limage. Paris: Minuit, 1990.

Fédida, Pierre. L'Absence. Paris: Gallimard, 1978.

Freud, Sigmund. "Ephémère destinée", Résultats, idées, problèmes, tome I. Paris: P.U.F. (1988), p. 233-236.

Freud, Sigmund. "La Négation", Résultats, idées, problèmes, tome II. Paris: P.U.F. (1987), p.135-139.

Freud, Sigmund. «Deuil et Mélancolie», Métapsychologie. Paris: Gallimard (1983), p. $148-173$. 
Kleinmann, Arthur et Byron Good (direction). Culture and Depression. Berkely/Los Angeles: University of California Press, 1985.

Klibansky, Raymond et al. Saturne et la Mélancolie. Paris: Gallimard, 1989.

Kofman, Sarah. Mélancolie de l'art. Paris: Galilée, 1985.

Kristeva, Julia. Soleil noir. Dépression et mélancolie. Paris: Gallimard, 1987.

Kristeva, Julia. "Fantasme et cinéma", La Révolte intime. Pouvoirs et limites de la psychanalyse, tome II. Paris: Fayard (1997), p. 118-151.

Lacan, Jacques. "Les quatre concepts fondamentaux de la psychanalyse", Le Séminaire, livre XI. Paris: Seuil, 1973.

Lambotte, Marie-Claude. Esthétique de la mélancolie. Paris: Éditions AubierMontaigne, 1984.

Merleau-Ponty, Maurice. L'CEil et l'esprit. Paris: Gallimard, 1964.

Metz, Christian. Le Signifiant imaginaire. Psychanalyse et cinéma. Paris: Union générale d'éditions, 1977.

Rosolato, Guy. "Le négatif et son lexique", Le Négatif. Figures et modalités (collectif). Paris: Dunod (1989), p. 9-23.

Roudinesco, Élisabeth et Michel Plon. Dictionnairede la psychanalyse. Paris: Fayard, 1997. 